\title{
Sensory profile, ferritin and zinc levels in preschool-aged children with symptoms of attention deficit hyperactivity disorder
}

\author{
Tuba Çelen Yoldaş ${ }^{1 \oplus}$, Meral Huri $^{2 \oplus}$, Hülya Kayıhann ${ }^{2 \oplus}$, Jale Karakaya ${ }^{3 \oplus}$, \\ Elif N. Özmert ${ }^{1 \oplus}$ \\ ${ }^{1}$ Division of Developmental Pediatrics, Department of Pediatrics, ${ }^{3}$ Department of Biostatistics, Hacettepe University Faculty of \\ Medicine, ${ }^{2}$ Occupational Therapy Unit, Hacettepe University Faculty of Health Sciences Ankara, Turkey.
}

\begin{abstract}
Background. Attention Deficit Hyperactivity Disorder (ADHD) is one of the most common neurodevelopmental disorders and has a big impact on the well-being of children. The disorder can lead to noticeable functional limitations for children and bio-ecological factors also contribute to symptoms of ADHD. We aimed to investigate the associations between ADHD symptoms and some related bio-ecological factors including serum ferritin, zinc levels and sensory processing in preschool-aged children.
\end{abstract}

Methods. Twenty-two children who had been referred to the division of Developmental Pediatrics because of ADHD symptoms and 22 participants from the general pediatric outpatient clinics were included in the study. The symptoms of ADHD were evaluated with Conners' Parent Rating Scale-Revised Short form. Complete blood count, serum ferritin and zinc levels were also evaluated. A blind occupational therapist implemented sensory processing measurements. The characteristics of each participant such as prematurity, perinatal complications, developmental practices and sociodemographic data were also considered.

Results. Sensory processing measurement analysis revealed that all Sensory Profile scores were significantly lower in the children with ADHD symptoms compared to the control group indicating that the child shows the behavior more than desired. The low level of zinc $(\mathrm{p}=0.026$, $\mathrm{OR}=6.153,95 \% \mathrm{CI}=1.247-30.362)$ and the presence of perinatal complications ( $\mathrm{p}=0.045$, $\mathrm{OR}=10.864,95 \% \mathrm{CI}=1.059-111.499)$ increased the risk of ADHD symptoms. We could not find an association for ferritin levels in our study.

Conclusions. The evaluation of zinc level and sensory profile parallel to other strategies can be recommended during the management of ADHD symptoms in preschool children.

Key words: attention deficit hyperactivity disorder, ferritin, sensory profile, zinc.

Attention Deficit Hyperactivity Disorder (ADHD) is one of the most common neurodevelopmental disorders and has a big impact on the well-being of children. ${ }^{1}$ In a recent national study ADHD has been reported as the most common mental disorder in children, with an overall prevalence of $19.5 \%$ without impairment, and $12.4 \%$ with impairment. ${ }^{2}$ The

$\triangle$ Tuba Çelen Yoldaş

tuba.celen@hacettepe.edu.tr

Received 12th February 2020, revised 27th April 2020, accepted 28th April 2020. disorder can lead to low quality of life and noticeable functional limitations for children and families. ${ }^{3}$ The presentation of the disorder is heterogeneous and the exact etiology remains unclear. ${ }^{4}$ Genetic polymorphism of dopamine transporter and receptors play an important role in the pathophysiology of the disorder. ${ }^{5,6}$ Bio-ecological factors also contribute to symptoms of ADHD. ${ }^{7-9}$ Iron and zinc metabolism are important factors that have commonly been investigated related to ADHD symptoms. ${ }^{10-17}$ Iron is necessary for normal myelination and neurotransmitter function in early brain development and is stored safely in 
the body as ferritin. ${ }^{11}$ A number of studies have indicated that children with ADHD have lower serum ferritin levels than children developing normally. ${ }^{12}$ Furthermore, low serum ferritin levels have been associated with symptom severity of ADHD. ${ }^{13}$ Likewise zinc levels and their association with symptom severity of ADHD have been investigated previously in school aged children. ${ }^{14}$ Zinc is a dopamine transporter with inhibitor properties that are related to stimulant medication therapies. ${ }^{15} \mathrm{It}$ has been shown that there might be different information processing in patients with zinc deficient ADHD and zinc supplementation might decrease the symptom severity of ADHD. ${ }^{16,17}$ Furthermore there are few studies investigating the association between ADHD symptoms and both iron and zinc metabolism but to our knowledge, there is no study investigating this association in preschool-aged children. ${ }^{10}$ It is noteworthy due to the importance of both zinc and iron in the dopamine pathway during this critical period.

In addition, children of these ages face multiple stimuli from their environments and may have difficulties processing them. A significant overlap has been shown for ADHD and sensory processing disorders in the literature. ${ }^{18}$ Sensory integration has been considered as a framework to handle and process sensory inputs from the environment. ${ }^{19}$ Sensory processing is defined as the ability to integrate multiple sensory information received from an individual's environment and to create adaptive responses to the sensory environment in order to provide meaningful participation in the activities of daily living. Although a relationship has been suggested between ADHD and sensory processing disorders in the literature, there is little data investigating the co-occurrence of these problems. ${ }^{20-23}$ It has been reported that in one of six children with ADHD there were sensory symptoms sufficient to adversely affect participation in daily life. ${ }^{21}$ Some researchers have noted that children with sensory processing deficits have more inattention, impulsivity, over-arousal and distractibility than children with normal sensory processing. ${ }^{20}$ In only one study with a sample of preschoolers was it shown that children with higher sensory deficits have displayed increased hyperactivity behavior. ${ }^{22}$ Other studies were mostly in school children and they found that children with ADHD had more sensory processing problems than those without ADHD. ${ }^{20,23}$ Although these limited studies have indicated an association between ADHD and sensory problems, further studies are needed to evaluate the potential impact of sensory processing for children with ADHD symptoms. ${ }^{20}$

In this study, we aimed to investigate the associations between ADHD symptoms and some related bio-ecological factors including serum ferritin, zinc levels and sensory processing in preschool-aged children. Since many environmental factors have been related to ADHD symptoms in this age group such as prematurity, perinatal complications, developmental practices, sociodemographic characteristics, these possible factors were also addressed. Little data exists about these problems in early childhood. We hypothesized that behavioral symptoms of ADHD are related to low serum ferritin and zinc levels, and sensory deficits are higher in children with ADHD symptoms. This is the first study to research all these bio-ecological factors in the same sample group, and particularly in preschool-aged children who are in a special sensitive period for brain development.

\section{Material and Methods}

\section{Procedures and Participants}

This cross-sectional study was conducted by the Division of Developmental Pediatrics, Department of Pediatrics between September 2016 and February 2017. After receiving ethical approval (GO 16/566), from the Ethical Committee of Hacettepe University Faculty of Medicine, 22 children who had been referred to the developmental pediatrics division because of ADHD symptoms, were included 
in the study. The comparison group included 22 participants who had been admitted to the general pediatric outpatient clinics for wellchild visits and similar in age and gender to the children with ADHD symptoms. Each child was examined by a pediatrician and screened for eligibility for the study. Inclusion criteria for the study were having normal development. After receiving written consent from the parent/ guardian of each participant they were referred to the division of developmental pediatrics. The general development and behavioral symptoms of each child were evaluated by the developmental pediatrician with the Denver II- Developmental Screening Test ${ }^{24}$ and Conners' Parent Rating Scale-Revised Short form. ${ }^{25}$ Symptoms of ADHD were evaluated by Conners' and children without developmental delay with Denver II were included in the study.

Blood sampling was conducted for the analysis of complete blood count, serum zinc and serum ferritin levels. The complete blood count was performed with the Coulter hematology analyzer. Serum ferritin and zinc levels were analyzed by D-mode spectrophotometric analysis. Serum levels of ferritin and zinc were categorized as low and normal according to laboratory cut-offs. All assessments were implemented at the first appointment. A blind occupational therapist implemented sensory processing measurements in a few days.

\section{Evaluation tools}

The questionnaire for prematurity, perinatal complications, developmental practices and sociodemographic characteristics was prepared by the study team. Developmental practices were playing with the child (regular or not), reading to the child (regular or not) and screen viewing of the child (mean time/ day). The meaning of 'regular' was accepted as at least once a day here. The questionnaire also encompassed mothers' social support dichotomizing as 'have' or 'not'. The number of children in the family and preschool education was also queried. Mothers were asked about perinatal complications whether they had a problem during pregnancy or delivery and, whether the baby had any postnatal medical problems (e.g., low birth weight, severe jaundice, seizures, difficulty breathing, or hernia) and was transferred to the intensive care unit. When the mothers answered yes for at least one of these questions the patient was accepted as having perinatal complications.

Hollingshead Redlich Scale which was based on the profession and training of both parents was used to determine social economic status. ${ }^{26}$

Denver-II- Developmental Screening Test is used to evaluate the developmental screening of 0 to 6-year-old children. ${ }^{27}$ Multiple translations have been conducted and all demonstrated cross-cultural reliability and validity. The original test was revised in 1990, forming the Denver II- Developmental Screening Test and the Turkish version was used in this study. ${ }^{24}$

Conners' Parent Rating Scale-Revised Short form evaluates the behavior of children as assessed by their parents. The scale includes 4 domains including oppositional behavior, inattentiveness, hyperactivity and ADHD index. Higher scores indicate higher symptom severity. The Turkish translation has good reliability and validity, Cronbach alpha and split-half reliability coefficients of the subscales were between 0.73 and 0.86 and 0.72 and 0.85 , respectively. Test-retest reliability coefficients of the subscales were between 0.56 and $0.72 .{ }^{25}$

The Sensory Profile (SP) is an occupational therapist guided caregiver questionnaire that determine how children between 3 and 10 years process sensory information in everyday situations with 125 items in which parents report the frequency of their child's response to items. ${ }^{28}$ The frequency is determined according to a Likert scale ( 1 = always: when the child responds in the manner $100 \%$ of the time and 5 =never). The rating "always" received the lowest score for each item because more frequent use of the behavior is undesirable. The cross-cultural adaptation of the questionnaire was carried out 
and Cronbach's alpha ranged from 0.63 to 0.97 with excellent test-/re-test reliability. ${ }^{29}$

Sensory Integration and Praxis Test (SIPT) is a standardized test battery which evaluates sensory processing disorders in children aged between 4 years and 8 years and 11 months. ${ }^{30}$ The test has high interrater reliability $\left(\mathrm{r}^{3} .90\right)$ and discriminates between typical and atypical samples $(\mathrm{p}<.01)$. The battery can be applied by a certified therapist and test-/re-test reliability is high. The score is evaluated by calculating the $\mathrm{Z}$ scores in a computer environment and interpreting the results by deducing the sensory profile of the child. ${ }^{31}$

\section{Data analysis}

Parametric tests were used for data with normal distributions whereas non-parametric tests were used otherwise. The normality for numerical variables was checked by using Kolmogorov-Smirnov test. Descriptive statistics were expressed as mean \pm standard deviation or median (min-max) according to the assumption of normal distribution. For categorical variables, count and percentages were used for description. Independent samples t-test was performed to compare the means of two groups for normal distribution data. Non-normal data were analyzed by using Mann-Whitney U-test. Pearson Chi-square test, Yates' Chi-square test or Fisher Exact tests were used to examine the difference between groups for categorical variables.

The relationship between symptoms of ADHD and sensory processing measurements was analyzed using the Spearman correlation coefficient.

Logistic regression analysis was performed to determine important risk factors for symptoms of ADHD. Factors determined by $p<0.20$ significance level in univariate analysis were included in the model. Using the logistic models, odds ratios (OR) and their respective 95\% confidence intervals (CI) were calculated. The backward stepwise elimination method was used to select significant factors. The power analysis of the study was made with NCSS 2007 in PASS programme to compare the groups according to Conners' scores and obtained 93\% of power. Statistical analysis was performed using SPSS 21 software and $p<0.05$ was accepted as significant.

\section{Results}

Forty-four participants were included in the study, 22 of them were children with ADHD symptoms. The mean ages of the case (children with ADHD symptoms) and control (children without ADHD symptoms) groups were $55.3 \pm$ 7.5 months and $54.0 \pm 8.1$ months, respectively with a range of 48-72 months. There were no differences in sociodemographic characteristics between the case and control groups ( $p>0.05)$, however perinatal complications including pregnancy, delivery and neonatal complications were higher in the case group than in the control group $(p=0.039)$. Detailed sociodemographic data of both groups are shown in Table I. The mean scores of all Conners' subscales; Oppositional ( $8.2 \pm 4.3$ by comparison $3.5 \pm 3.2)$, Cognitive Problems-Inattention (6.8 \pm 4.6 by comparison $1.0 \pm 1.4)$, Hyperactivity $(8.9 \pm 4.2$ by comparison $1.9 \pm 2.9)$, ADHD index scales (18.6 \pm 7.3 by comparison $3.7 \pm 3.9$ ) were significantly higher in the case group than in the control group ( $\mathrm{p}<0.001$ for all comparisons).

Complete blood analyses were usually in the normal range and similar in the case and control groups ( $p=0.463)$. Children with low zinc levels were higher in the case group $(n=9, \% 40.9)$ than in the control group $(n=3, \% 13.6)(p=0.042)$. Mostly children in the case $(n=14,63.6 \%)$ and the control $(n=13,59.1 \%)$ group had normal ferritin levels, and there was no significant difference between the groups $(p=0.757)$.

Sensory processing measurement analysis revealed that all SP scores were significantly lower in the case group compared to the control group indicating that the child shows the behavior more than desired (Table II). 
Table I. Sociodemographic characteristics of the case (children with ADHD symptoms) and control (children without ADHD symptoms) groups.

\begin{tabular}{lccc}
\hline Sociodemographic characteristic & Case & Control & $\mathrm{p}$ \\
$\mathrm{N}(\%)$ & $\mathrm{n}=22$ & $\mathrm{n}=22$ & 0.455 \\
\hline Age (mean \pm SD) (month) & $55.3 \pm 7.5$ & $54.0 \pm 8.1$ & 0.186 \\
Male & $18(81.8)$ & $13(59.1)$ & 0.150 \\
Preterm & $7(31.8)$ & $3(13.6)$ & 0.039 \\
Perinatal complications & $6(27.3)$ & $1(4.5)$ & 0.105 \\
Maternal age (mean \pm SD) & $33.2 \pm 4.4$ & $30.8 \pm 5.2$ & 0.221 \\
Maternal education (under high school) & $10(45.4)$ & $10(45.5)$ & 1.000 \\
Maternal employment (housewife) & $18(81.8)$ & $19(86.4)$ & 0.565 \\
Paternal age (mean \pm SD) & $35.8 \pm 4.04$ & $34.9 \pm 5.6$ & 0.267 \\
Paternal education (under high school) & $8(36.4)$ & $7(31.8)$ & 0.457 \\
Paternal employment (regular) & $19(86.4)$ & $16(72.7)$ & 1.000 \\
Married & $21(95.5)$ & $22(100)$ & 0.632 \\
Social support (have) & $8(38.1)$ & $11(50)$ & 0.747 \\
Reading book to the child (regularly) & $7(31.8)$ & $5(22.7)$ & 0.570 \\
Screen viewing of the child (mean $\pm S D)(h o u r s)$ & $4.11 \pm 2.58$ & $3.77 \pm 2.72$ & 0.252 \\
Playing with the child (regularly) & $7(31.8)$ & $7(31.8)$ & 0.223 \\
Preschool education & $12(54.5)$ & $7(31.8)$ & 0.478 \\
Number of children (median)(range) & $2(1-4)$ & $2(1-3)$ & 0.806 \\
SES(mean \pm SD) & $3.09 \pm 1.15$ & $3.09 \pm 0.81$ & \\
\hline
\end{tabular}

ADHD: attention deficit hyperactivity disorder, SD: standard deviations, SES: social economic status.

Table II. Sensory profile test results of the groups.

\begin{tabular}{lcc}
\hline Sensory profile test* $($ mean \pm SD) & $\begin{array}{c}\text { Case group** } \\
\text { (with ADHD symptoms) }\end{array}$ & $\begin{array}{c}\text { Control group** } \\
\text { (without ADHD symptoms) }\end{array}$ \\
\hline Auditory processing & $20.634 \pm 4.945$ & $31.436 \pm 5.043$ \\
Visual processing & $27.832 \pm 4.843$ & $34.435 \pm 5.265$ \\
Vestibular processing & $39.376 \pm 5.769$ & $48.459 \pm 3.634$ \\
Touch processing & $61.471 \pm 12.738$ & $79.376 \pm 6.487$ \\
Multisensory processing & $19.482 \pm 2.487$ & $28.582 \pm 3.256$ \\
Oral processing & $42.634 \pm 7.698$ & $50.671 \pm 6.112$ \\
Sensory Processing related to tone endurance/tone & $34.132 \pm 5.231$ & $43.532 \pm 4.523$ \\
Modulation related to body position and movement & $32.271 \pm 5.482$ & $42.612 \pm 4.287$ \\
Modulation of movement affecting activity level & $18.375 \pm 3.571$ & $24.438 \pm 4.217$ \\
Modulation of sensory input affecting emotion & $12.459 \pm 2.641$ & $16.437 \pm 2.653$ \\
Modulation of visual input affecting emotion & $10.582 \pm 2.781$ & $14.659 \pm 2.398$ \\
Emotional social responses & $50.247 \pm 9.621$ & $69.351 \pm 9.430$ \\
Behavioral outcomes of sensory processing & $16.471 \pm 3.874$ & $22.472 \pm 2.738$ \\
Items indicating threshold of response & $10.217 \pm 3.211$ & $12.935 \pm 2.261$ \\
\hline
\end{tabular}

${ }^{*}$ Higher scores indicate better scores according to Likert scale, ${ }^{* *}: \mathrm{p}<0.05$ for all comparisons of Sensory Profile Test scores between the case (with ADHD symptoms) and control (without ADHD symptoms) groups, SD: Standard Deviation 
Similarly, some of the SIPT sub scores of the case group were significantly lower than in the control group (Table III). There was no clinically significant association between SP, SIPT and ADHD index scores in correlation analysis $(p>0.05)$. Also, there was no association between low ferritin levels and SP and SIPT in either group ( $p>0.05$ ). However low zinc levels were associated with the 'Modulation of movement affecting the activity level ( $p=0.033)$ of SP and the 'Manual form perception' ( $p=0.001$ ) of SPIT in the case group.

Step-wise logistic regression analyses were also implemented to identify the risk factors associated with the symptoms of ADHD. In multivariate analysis, factors determined by $\mathrm{p}<0.20$ significance level in univariate analysis (child gender, maternal age, perinatal complications, zinc and prematurity) were included in the model. Finally, low zinc level ( $p=$ 0.026, $\mathrm{OR}=6.153,95 \% \mathrm{CI}=1.247-30.362)$ and the presence of perinatal complications $(\mathrm{p}=0.045$,
$\mathrm{OR}=10.864,95 \% \mathrm{CI}=1.059-111.499)$ increased the risk of the symptoms of the disorder.

\section{Discussion}

In this study, we identified higher sensory deficits in children with symptoms of ADHD and, among all possible related factors low zinc levels and perinatal complications were found to be associated with symptoms of ADHD. To the best of our knowledge, this is the first report discussing these bio-ecological factors in the same-sample group and also in preschool children.

Sensory processing measurements of all participants wereevaluated in the study. Sensory Profile scores of the children with ADHD symptoms were lower than the control group. A theoretical overlap has been noted between ADHD and sensory processing disorder in the literature. ${ }^{18}$ However to our knowledge there is only one study with a sample of preschoolers

Table III. Sensory integration and praxis test (SIPT) scores of the groups.

\begin{tabular}{lcc}
\hline SIPT tests (mean \pm SD) & $\begin{array}{c}\text { Case group } \\
\text { (with ADHD symptoms) }\end{array}$ & $\begin{array}{c}\text { Control group } \\
\text { (without ADHD symptoms) }\end{array}$ \\
\hline Space visualization & $-0.92 \pm 0.91^{*}$ & $-0.59 \pm 0.78^{*}$ \\
Figure-ground perception & $-0.59 \pm 1.21$ & $-0.29 \pm 1.01$ \\
Standing and walking balance & $-1.74 \pm 1.32^{*}$ & $-1.32 \pm 1.24^{*}$ \\
Design copy & $-1.88 \pm 1.59^{*}$ & $-1.39 \pm 1.49^{*}$ \\
Postural praxis & $-1.52 \pm 1.39^{*}$ & $-1.21 \pm 1.42^{*}$ \\
Bilateral motor coordination & $-1.12 \pm 1.12$ & $-1.81 \pm 1.04$ \\
Praxis on verbal command & $-1.46 \pm 1.87$ & $-1.93 \pm 1.76$ \\
Constructional praxis & $-1.15 \pm 1.39$ & $-1.66 \pm 1.42$ \\
Post-rotary nystagmus & $-0.72 \pm 1.27^{*}$ & $-0.26 \pm 1.32^{*}$ \\
Motor accuracy & $-0.73 \pm 1.29 *$ & $-0.31 \pm 1.28^{*}$ \\
Sequencing praxis & $-1.23 \pm 1.14$ & $-0.88 \pm 1.02$ \\
Oral praxis & $-1.59 \pm 1.51$ & $-1.11 \pm 1.23$ \\
Manual form perception & $-1.24 \pm 1.59$ & $-0.81 \pm 1.52$ \\
Kinesthesia & $-1.43 \pm 1.71$ & $-1.12 \pm 1.64$ \\
Finder identification & $-0.99 \pm 1.21$ & $-0.61 \pm 1.19$ \\
Graphestesia & $-1.44 \pm 1.29$ & $-1.12 \pm 1.21$ \\
Localization of tactile stimuli & $-0.59 \pm 1.93$ & $-0.53 \pm 1.62$
\end{tabular}

SIPT: Sensory Integration and Praxis Test, ${ }^{*}: p<0.05$ for the scores of SIPT between the case (with ADHD symptoms) and control (without ADHD symptoms) groups, SD: Standard Deviation 
and it has demonstrated that children with more sensory deficits have displayed increased hyperactivity behavior. ${ }^{22}$ Other limited studies in school-aged children have also reported that children with ADHD symptoms had more sensory processing problems than those without ADHD symptoms. ${ }^{20,21,23}$ Ultimately they were all consistent with the findings of this study. Ferritin and zinc levels were also assessed in relation to the SP and SIPT scores which we did not come across in current literature. There was an association between low zinc levels and certain subgroups of SP and SIPT in the children with ADHD symptoms. However, further studies are needed to understand the underlying mechanism in this field.

An important factor associated with symptoms of ADHD in our study was low zinc levels. Researchers have begun to look at all possibilities in the field of nutrition to better understand and address mental health issues. Noticeable evidence for the role of micronutrients in mental health has come from those studies focusing on the role of zinc in common disorders such as ADHD. Lower zinc concentrations have been found in children with ADHD in several studies with the suggestion that zinc levels might be related to the severity of symptoms. ${ }^{16} \mathrm{~A}$ review with numerous controlled studies has reported cross-sectional evidence of lower zinc tissue concentrations in children with ADHD than in control subjects and compared with population norms. ${ }^{32}$ Consistent with these studies, it was found that low-level zinc increased the risk of having ADHD symptoms.

Ferritin is another widely investigated factor in this field. Lower serum ferritin levels in children with ADHD comparing to children with normal development and, the associations between low ferritin levels and symptom severity have been reported previously..$^{10-13}$ Similarly, a recent meta-analysis has indicated that children with ADHD have lower serum ferritin levels than children developing typically. ${ }^{7}$ Although these reports, we could not find an association for ferritin levels in our study. However, the previous studies were mostly in school-aged children. There need to be further studies with larger samples in preschool-aged children.

Another factor associated with symptoms of ADHD was perinatal complications among the results. Prematurity was higher in children with ADHD symptoms, but there was no significant difference between the groups. Despite a clear demonstration of the association between prematurity and ADHD, there remains insufficient evidence to support a definite causal relationship. ${ }^{33-35}$ It might be biologically plausible that as there is less time for neural development, prematurity is a risk factor of ADHD; however it is more likely that the underlying mechanism operates through a variety of factors such as increased incidence of obstetric complications which may lead to neural insult. ${ }^{36}$ Consistent with literature our regression analysis revealed an association between perinatal complications and symptoms of ADHD. There has been a considerable amount of investigation researching the association of perinatal complications including pregnancy, labour/delivery and neonatal complications with ADHD. It has been reported that perinatal complications might contribute to symptoms of ADHD, because of the immaturity of the central nervous system and the vulnerability of functional abnormalities in neurological pathways. ${ }^{37}$

Behavioral symptoms of ADHD were evaluated in this study, it was not a diagnostic process. The small sample size was an important limitation of the study. However, the power analysis of the study has demonstrated that the sample was large enough to enable to compare the groups according to Conners' scores. Sensorial evaluations were made by a blind occupational therapist which we believe is an important strength of the study. It was noteworthy to implement all these measurements in the samesample to contribute to literature related to preschool children with ADHD symptoms.

In conclusion, several bio-ecological factors have been investigated related to ADHD symptoms in this study. Our results demonstrate that low 
zinc levels and perinatal complications were related to symptoms of ADHD and, sensory deficits were determined more in children with ADHD symptoms. We could not find an association with ferritin levels in our study sample. The evaluation of zinc level and sensory profile parallel to other strategies can be recommended during the management of ADHD symptoms in preschool children.

\section{Acknowledgement}

We would like to thank the families for participating in this study.

\section{REFERENCES}

1. Wolraich M, Brown L, Brown RT, et al; Subcommittee on Attention-Deficit/Hyperactivity Disorder; Steering Committee on Quality Improvement and Management. ADHD: clinical practice guideline for the diagnosis, evaluation, and treatment of attention-deficit/hyperactivity disorder in children and adolescents. Pediatrics 2011; 128: 1007-1022.

2. Ercan ES, Polanczyk G, Akyol Ardic U, et al. The prevalence of childhood psychopathology in Turkey: a cross-sectional multicenter nationwide study (EPICPAT-T). Nord J Psychiatry 2019; 73: 132-140.

3. Thapar A, Cooper M. Attention deficit hyperactivity disorder. Lancet 2016; 387: 1240-1250.

4. Tandon M, Pergjika A. Attention deficit hyperactivity disorder in preschool-age children. Child Adolesc Psychiatr Clin N Am 2017; 26: 523-538.

5. Faraone SV, Perlis RH, Doyle AE, et al. Molecular genetics of attention-deficit/hyperactivity disorder. Biol Psychiatry 2005; 57: 1313-1323.

6. Biederman J. Attention-deficit/hyperactivity disorder: a selective overview. Biol Psychiatry 2005; 57: 12151220 .

7. Tseng PT, Cheng YS, Yen CF, et al. Peripheral iron levels in children with attention-deficit hyperactivity disorder: a systematic review and meta-analysis. Sci Rep 2018; 8: 788.

8. Konofal E, Lecendreux M, Deron J, et al. Effects of iron supplementation on attention deficit hyperactivity disorder in children. Pediatr Neurol 2008; 38: 20-26.

9. Verlaet AA, Noriega DB, Hermans N, Savelkoul HF. Nutrition, immunological mechanisms and dietary immunomodulation in ADHD. Eur Child Adolesc Psychiatry 2014; 23: 519-529.
10. Oner $\mathrm{O}$, Oner $\mathrm{P}$, Bozkurt $\mathrm{OH}$, et al. Effects of zinc and ferritin levels on parent and teacher reported symptom scores in attention deficit hyperactivity disorder. Child Psychiatry Hum Dev 2010; 41: 441447.

11. Doom JR, Georgieff MK. Striking while the iron is hot: Understanding the biological and neurodevelopmental effects of iron deficiency to optimize intervention in early childhood. Curr Pediatr Rep 2014; 2: 291-298.

12. Wang Y, Huang L, Zhang L, Qu Y, Mu D. Iron status in attention-deficit/hyperactivity disorder: a systematic review and meta-analysis. PLoS One 2017; 12: e0169145.

13. Konofal E, Lecendreux M, Arnulf I, Mouren MC. Iron deficiency in children with attention-deficit/ hyperactivity disorder. Arch Pediatr Adolesc Med 2004; 158: 1113-1115.

14. Arnold LE, Bozzolo H, Hollway J, et al. Serum zinc correlates with parent- and teacher- rated inattention in children with attention-deficit/hyperactivity disorder. J Child Adolesc Psychopharmacol 2005; 15: 628-636.

15. Krause J. SPECT and PET of the dopamine transporter in attention-deficit/hyperactivity disorder. Expert Rev Neurother 2008; 8: 611-625.

16. Yorbik O, Ozdag MF, Olgun A, Senol MG, Bek S, Akman S. Potential effects of zinc on information processing in boys with attention deficit hyperactivity disorder. Prog Neuropsychopharmacol Biol Psychiatry 2008; 32: 662-667.

17. Uckardes Y, Ozmert EN, Unal F, Yurdakok K. Effects of zinc supplementation on parent and teacher behaviour rating scores in low socioeconomic level Turkish primary school children. Acta Paediatr 2009; 98: 731-736.

18. Koziol LF, Budding D. ADHD and sensory processing disorders: placing the diagnostic issues in context. Appl Neuropsychol Child 2012; 1: 137144.

19. Zimmer M, Desch L. Section on Complementary and Integrative Medicine, Council on Children with Disabilities; American Academy of Pediatrics. Sensory integration therapies for children with developmental and behavioral disorders. Pediatrics 2012; 129: 1186-1189.

20. Pfeiffer B, Daly BP, Nicholls EG, Gullo DF. Assessing sensory processing problems in children with and without attention deficit hyperactivity disorder. Phys Occup Ther Pediatr 2015; 35: 1-12.

21. Ben-Sasson A, Carter AS, Briggs-Gowan MJ. Sensory over-responsivity in elementary school: prevalence and social-emotional correlates. J Abnorm Child 
Psychol 2009; 37: 705-716.

22. Yochman A, Parush S, Ornoy A. Responses of preschool children with and without ADHD to sensory events in daily life. Am J Occup Ther 2004; 58: 294-302.

23. Engel-Yeger B, Ziv-On D. The relationship between sensory processing difficulties and leisure activity preference of children with different types of ADHD. Res Dev Disabil 2011; 32: 1154-1162.

24. Durmazlar N, Ozturk C, Ural B, Karaagaoglu E, Anlar B. Turkish children's performance on Denver II: effect of sex and mother's education. Dev Med Child Neurol 1998; 40: 411-416.

25. Kaner S, Büyüköztürk, Ş, İşeri, E. Conners anababa dereceleme ölçeği-yenilenmiş kısa: Türkiye stardardizasyon çalışması. Nöropsikiyatri Arşivi 2013; 50: 100-109.

26. Hollingshead AB, Redlich FC. Social class and mental illness: a community study. 1958. Am J Public Health 2007; 97: 1756-1757.

27. Frankenburg WK, Dodds J, Archer P, Shapiro H, Bresnick B. The Denver II: a major revision and restandardization of the Denver Developmental Screening Test. Pediatrics 1992; 89: 91-97.

28. Ermer J, Dunn W. The sensory profile: a discriminant analysis of children with and without disabilities. Am J Occup Ther 1998; 52: 283-290.

29. Kayihan H, Akel BS, Salar S, et al. Development of a Turkish version of the sensory profile: translation, cross-cultural adaptation, and psychometric validation. Percept Mot Skills 2015; 120: 971-986.

30. Ayres AJ. Sensory Integration and Praxis Test (SIPT). Los Angeles: Western Psychological Services, 1989.

31. Schaaf RC, Burke JP, Cohn E, et al. State of measurement in occupational therapy using sensory integration. Am J Occup Ther 2014; 68: e149-e153.

32. DiGirolamo AM, Ramirez-Zea M. Role of zinc in maternal and child mental health. Am J Clin Nutr 2009; 89: S940-S945.

33. Henriksen L, Wu CS, Secher NJ, Obel C, Juhl M. Medical augmentation of labor and the risk of ADHD in offspring: a population-based study. Pediatrics 2015; 135: e672-e677.

34. Silva D, Colvin L, Hagemann E, Bower C. Environmental risk factors by gender associated with attention-deficit/hyperactivity disorder. Pediatrics 2014; 133: e14-e22.

35. Halmoy A, Klungsoyr K, Skjaerven R, Haavik J. Preand perinatal risk factors in adults with attentiondeficit/hyperactivity disorder. Biol Psychiatry 2012; 71: 474-481.

36. Sciberras E, Mulraney M, Silva D, Coghill D. Prenatal risk factors and the etiology of ADHD-review of existing evidence. Curr Psychiatry Rep 2017; 19: 1.

37. Ketzer CR, Gallois C, Martinez AL, Rohde LA, Schmitz M. Is there an association between perinatal complications and attention-deficit/ hyperactivity disorder-inattentive type in children and adolescents? Braz J Psychiatry 2012; 34: 321-328. 